\title{
Performance Metrics for Segmentation Algorithms in Brain MRI for Early Detection of Autism
}

\author{
Nagashree N, Premjyoti Patil, Shantakumar Patil, Mallikarjun Kokatanur
}

\begin{abstract}
Autism is an abnormal condition of human brain neurons, which makes individuals attention deficient, unable to speak and several other neurodevelopmental disorders as detected in the children with the age group of 2 to 5 years. However, autism is a neurological irregularity with more than one behavioral problem. Autism would be generally detected by behavioral symptoms, but early detection was not possible with behavioral approach. So, studying the structure of brain by using MRI image of the brain would be an efficient technique in early detection of autism. Various image classification and segmentation methods have been developed by many researchers. This work proposes a new performance metrics to find out efficiency of segmentation algorithms.
\end{abstract}

Keywords: ASD, Genetic Threshold, K-means, Segmentation.

\section{INTRODUCTION}

Autism is a serious developmental disorder which affects the child's ability to interact and behave. The neuroanatomy of autism is very difficult to analyze. Autism shows abnormalities in all the 4 regions of brain viz, parietal sector, frontal sector, occipital sector and lastly temporal sector. Abnormalities in the autistic brain can also affect in cerebellum, sensory portions of brain and corpus collosum, where the left and right hemispheres of the brain meet using axons. There is already an evidence that, the change in structure of cerebellum has directly related to neurological disorders. Genomic features have also been found out in this case. Autism can generally be detected by expert using behavioral therapy by interacting with the individuals specially in the age group of 2 and 7. But there is a need for very early detection of autism before the age of 1 or 2 . That can be possible only by imaging studies and tests like CT scans, PET scans or MRI and fMRI imaging. Main challenge in Autism detection lies in the accuracy of the feature points extraction and once extraction done, how to classify.

Revised Manuscript Received on December 15, 2019

Nagashree N*, VTU Research Scholar, Dept of CSE, Nagarjuna College of Engineering \& Technology, Bengaluru, India. Email: astrodamsel@gmail.com

Dr. Premjyoti Patil, Professor, Dept of E\&C, Nagarjuna College of Engineering \& Technology, Bengaluru, India. Email: premjyoti.patil@ncetmail.com

Dr. Shantakumar Patil, Professor, Dept of CSE, Nagarjuna College of Engineering \& Technology, Bengaluru, India. Email: shantakumar@ncetmail.com

Mr. Mallikarjun Kokatanur, Senior Software Engineer, Sabre Travel Technologies Pvt. Ltd, Bengaluru, India. Email: mallubk@gmail.com
There are many efficient segmentation algorithms available to extract the feature points and further various machine algorithms are researched in classifying feature points for efficient detection of autism in early age. Another challenge lies in medical testing or diagnosis since autism is a behavioral related psychological factor rarely people go for medical imaging tests.

To detect the efficiency of the segmentation algorithms listed, there is a requirement to find out the metrics to detect the performance of each segmentation algorithm and that is the further part of this research paper.

The very first step in autism detection is pre-processing where the noise in the image is removed by various mathematical models. Next comes Image segmentation. It refers to the process of diving a digital image into multiple parts. The main aim of segmentation is to give different and clear view for the original image.

The segmentation of image results in partitioning or subset of main image. [1]. Therefore, medical image segmentation plays a significant role in clinical diagnosis. Generally medical imaging is very challenging task because they have poor resolution [2]. There are many medical imaging techniques to study the anatomy of human brain. Different methods are PET, CT, MRI, fMRI [3]. Since behavioral diagnosis of autism required lots of expert intervention and difficult to detect in early age there is a need for imaging methods [4]. So this would in fact require efficient segmentation methods with some selected properties of images [5]. Many image segmentation algorithms derives its parts based on two criteria: region based and intensity based [6]. In case of first method, the algorithm is based on partitioning the image on intensity basis and where as the later approach evaluates on coordinate area axis. The literature review says there are number of segmentation methods such as histogram based methods, edge-based methods, artificial neural network based segmentation methods, physical model based approaches, region-based methods (region splitting, growing, and merging), and clustering methods (Fuzzy C-means clustering, K-means clustering, Mean Shift, and Expectation Maximization) [7-9].Since image classification and segmentation plays a very important role in pattern matching for any given medical imaging methods, there is no universally accepted method for it. The efficiency of the algorithm depends on the kind of problem associated in the research. [10]. 


\section{METHODOLOGY}

The proposed method of brain MRI in autism detection includes the following steps:

\section{a) Dataset Representation}

In our proposed system we have made use of 500 brain MRI of autistic patients which we have collected from

\section{b) Pre-Processing}

Preprocessing is the technique of removing noises from the MRI images. Region based binary mask extraction: This method of mask extraction is carried out by dividing the whole image into several parts called regions. Region masking is done by selecting a threshold and there are two ways of doing this:

One is to find out max-min difference and the other is calculating average of blocks. Hence this technique is applied for brain making in original MRI data.

\section{c) Segmentation}

Segmentation is the extracting of region of interest from the original image. Following segmentation methods are implemented and compared:

\section{K means Clustering Algorithm}

K means clustering mathematical model:

$c a_{1} \cup c a_{2} \cup c a_{3} \ldots c a_{k}=p \sum_{i=1}^{k} \sum_{x \in c a_{i}} 1\left\|+-\frac{1}{|k i|} \sum_{t_{j} \in k_{j}} t_{j}\right\|^{2}$

The algorithm steps are as follows:

Step 1: Make clusters into one group called $\mathrm{k}$ groups and $\mathrm{k}$ is fixed.

Step 2: Select any clusters and find the centroid

Step 3: Fix the object to the nearest cluster centroid, Euclidian distance formula helps in finding the nearest node or cluster.

Step 4: Identify the cluster centroid of all objects, repeat steps 2-4 until, in continuous rounds identical object assigns to same clusters.

\section{FCM Segmentation Method:}

Mathematical model:

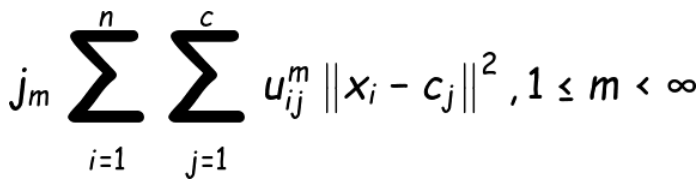

Algorithm:

Step 1: Initialize $\mathrm{p}=\left[\mathrm{P}_{\mathrm{ij}}\right]$ matrix

Step 2: In N-step: find the centers vectors $C\{K\}=\left[C_{j}\right]$ with $U$ ABIDE.

$$
c_{j}=\frac{\sum_{i=1}^{n} u_{i j}^{m} \cdot x_{i}}{\sum_{i=1}^{n} u_{i j}^{m}}
$$

Update $\mathrm{P}(\mathrm{K}), \mathrm{P}(\mathrm{K}-1)$.

Step 3: If
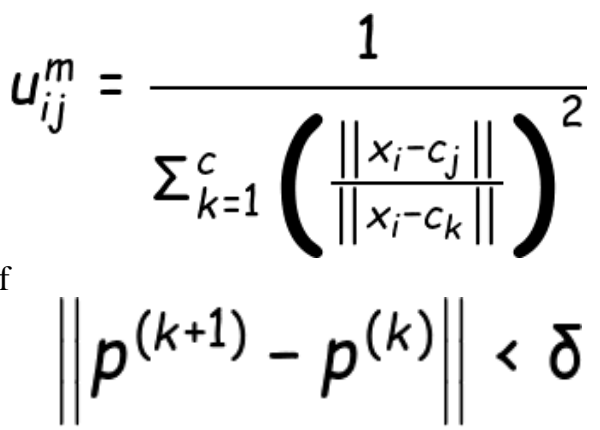

Terminate the process else go back to step 2 .

\section{Genetic Threshold Algorithm:}

The GN algorithm of populace amount begins with nth random size. Finally, it will select member with good size.

Pseudo code:

1. Read the image

2. Find out Image Histogram

3. Populate initial set of chromosomes

4. For $\mathrm{i}=0$ to number of iterations

5. While (size(pop) $>$ size (Newpop))

6. Select two chromosomes Ch1, Ch2.

7. If (rand $<$ crossover rate) then

8. Perform crossover Chnew1, chnew2

9. IF (rand <mutation rate) then

10. Mutate chnew 1

11. If (rand <mutation rate) then

12. Mutate chnew2

13. Add newpop.

\section{PERFORMANCE METRICS OF THE SEGMENTATION ALGORITHMS}

\section{Method 1: Pmax Metric}

In the segmentation of Brain MRI images, many factors are taken into consideration in efficient detection of segments.

Feature extraction is one such method to segment the image. The segmentation efficiency can be detected by finding the more features in any algorithm. Out of the methods selected for segmentation, a common mathematical model is proposed to analyze performance.

Step 1: Populate an array $A=\left\{a_{1}, a_{2}, a_{3}, \ldots \ldots \ldots\right\}$ where $a_{1}$, $a_{2}, \ldots \ldots$ are the set of coordinate points obtained out of segmentation algorithm.

Step 2: Calculate the area of the region from the coordinate points obtained

$$
\text { Area }_{\text {region }}=\int_{0}^{1} x d x
$$


Step 3: Find out the centroid of the coordinate points. Centroid can be calculated by taking 4 outermost coordinate points. Let $\mathrm{O}_{1}$ and $\mathrm{O}_{2}$ be the left and right outermost feature point and $\mathrm{O}_{3}$ and $\mathrm{O}_{4}$ are the top and bottom most features.

Calculate $\mathrm{d}_{1}=\left(\mathrm{O}_{1}+\mathrm{O}_{2}\right) / 2$ and

$$
\mathrm{d}_{2}=\left(\mathrm{O}_{3}+\mathrm{O}_{4}\right) / 2
$$

Centroid $\mathrm{C}=\mathrm{d}_{1}+\mathrm{d}_{2}$ store the value of $\mathrm{C}_{\mathrm{i}}$ for each MRI brain image.

Step 4: Find the threshold value by repeating step 2 and 3 .

Step 5: Find the distance between Centroid $C$ and each element in the array

$$
\begin{gathered}
A=\left\{a_{1}, a_{2}, a_{3}, \ldots \ldots \ldots\right\} . \\
\text { If } D<=\text { threshold, } D_{\text {in }}=\left\{a_{1}, a_{2}, \ldots .\right\} \\
\text { Else } \\
D_{\text {out }}=\left\{a_{1}, a_{2}, \ldots \ldots \ldots .\right\}
\end{gathered}
$$

Step 6: Find $N=$ count of $\left\{D_{\text {in }}\right\}$ for each algorithm i.e $N_{1}, N_{2}$ and $\mathrm{N}_{3}$ and

$$
\mathrm{N}_{\max }=\left\{\mathrm{N}_{1}, \mathrm{~N}_{2}, \mathrm{~N}_{3}\right\}
$$

Here $\mathrm{N}_{\max }$ is maximum no. of feature points falling within interested region.

\section{Method 2: Percentage Metric}

From the collected feature points calculate the percentage of autistic features against the normal feature points to get $f(x, y)$ where $x$ and $y$ are the coordinate points of feature points of segmented image

$$
f(x, y)=\frac{N_{(i) a u t i s t i c}}{N_{(i) n o r m a l}} \times 100
$$

\section{RESULTS AND DISCUSSION}

Brain has 2 hemispheres, left and the right. To interconnect

\begin{tabular}{|c|c|}
\hline Segmentation & Autism \\
\hline K-Means & \\
\hline $\mathrm{FCM}$ & \\
\hline Genetic Threshold & \\
\hline
\end{tabular}
these two hemispheres of brain, corpus collosum has 200 million axons. Each hemisphere of brain is meant for some specific functionality. For a better decision-making ability or to process some complex information, both hemispheres of the brain are required and there comes the importance of corpus collosum.

Figure 1: Corpus callosum of brain for autistic individual

But when corpus collosum is working properly, such people may behave abnormal. Physically those persons may be heathy, but they are mentally abnormal.

The Figure 1 shows the corpus collosum status of autistic and normal brain.
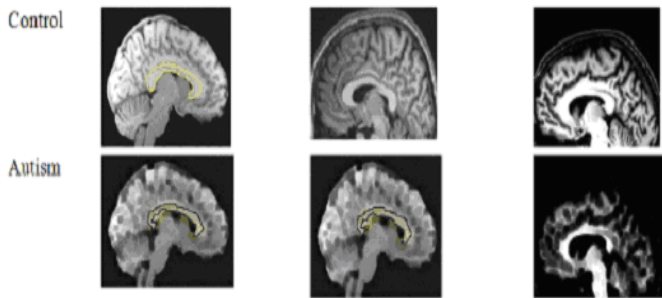

Figure 2: Gray matter identification of autistic and control individuals

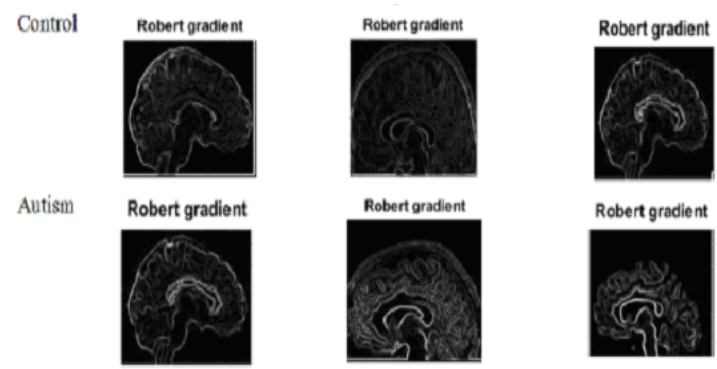

Figure 3: Edge detection of autistic and control individuals

\section{Calculate the Area of Corpus Callosum}

Once the segmented image is obtained, using binarization technique, the area of the corpus collosum is calculated for every feature point obtained.

Table 1. shows the performance metrics of proposed segmentation algorithm using binarization technique. The values are calculated for indicated area of corpus callosum. The calculated values are 396 pixels in case of normal brain and 253 pixels for autistic child's brain. From these calculated values, it is observed that the values of corpus callosum of autistic are reduced than normal.

Table 1: Comparison in Pixel numbers to distinguish between autistic and normal brain

\begin{tabular}{|l|l|l|}
\hline \multicolumn{1}{|c|}{$\begin{array}{c}\text { Area of } \\
\text { brain }\end{array}$} & Variants & $\begin{array}{c}\text { Performance metrics } \\
\text { in pixels }\end{array}$ \\
\hline $\begin{array}{l}\text { Corpus } \\
\text { Collosum }\end{array}$ & $\begin{array}{l}\text { Autistic } \\
\text { Brain }\end{array}$ & 253 \\
\hline $\begin{array}{l}\text { Corpus } \\
\text { Collosum }\end{array}$ & $\begin{array}{l}\text { Normal } \\
\text { Brain }\end{array}$ & 396 \\
\hline
\end{tabular}

Using the performance metrics discussed earlier, autistic brain features are reduced than the features of normal brain. Figure 4 shows the graphical representation of plotted feature points using the segmentation algorithm. 


\section{Performance Metrics for Segmentation Algorithms in Brain MRI for Early Detection of Autism}

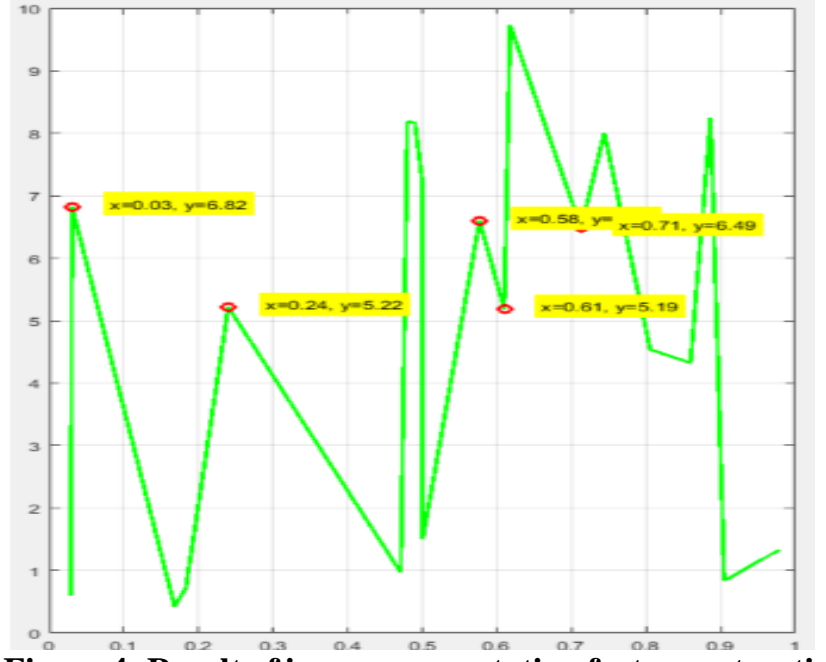

Figure 4: Result of image segmentation feature extraction represented as coordinate points

\section{REFERENCES}

1. B.J. Bipin Nair, T.R. Pruthvi, "A Segmentation Approaches to Detec Autism and Dementia from Brain MRI", International Journal of Recent Technology and Engineering (IJRTE) ISSN: 2277-3878, Volume-7, Issue-5C, February 2019.

2. Eman Abdel-Maksoud, Mohammed Elmogy, "Brain tumor segmentation based on a hybrid clustering technique", Egyptian Informatics Journal (2015) 16, 71-81.

3. IEEE journal of Radio frequency identification and analysis.

4. Mousumi Bala, Suraiya Yasmin, "Study the Corpus Callosum of Brain to Explore Autism Employing Image Segmentation", International Journal of Neuroscience and Behavioral Science 4(3): 37-44, 2016.

5. B.J. Bipin Nair, Gopi Krishna Ashok, N.R. Sreekumar, "Classification of Autism based on Feature Extraction from Segmented Brain MRI", International Journal of Recent Technology and Engineering (IJRTE).

6. Bram van den Bekerom, "Using Machine Learning for Detection of Autism Spectrum Disorder", 26th Twente Student Conference on IT Feb 3th, 2017, Enschede, The Netherlands.

7. Rajneesh Mahajan, Stewart H. Mostofsky, "Neuroimaging Endophenotypes in Autism Spectrum Disorder", HHS Public Access Author manuscript CNS Spectr. Author manuscript; available in PMC 2016 August 01 .

8. Hongyoon Choi, "Functional connectivity patterns of autism spectrum disorder identified by deep feature learning.".

9. Jared A Nielsen, Brandon A, "Abnormal lateralization of functional connectivity”, Nielsen et al. Molecular Autism 2014, 5:8, Open access journal.

10. Wojciech bienichi, "New image processing algorithms in computer vision systems for pathomorphologic diagnostics", Research gate, January 2019

11. Ayman El-Baz; Manuel F. Casanova; Georgy Gimel'farb; Meghan Mott “ A NEW IMAGE ANALYSIS APPROACH FOR AUTOMATIC CLASSIFICATION OF AUTISTIC BRAINS", 2007 4th IEEE International Symposium on Biomedical Imaging: From Nano to Macro

12. Heather CodyHazlettaMichele D.PoebGuidoGerigcRachel GimpelSmithaJosephPivena, "Cortical Gray and White Brain Tissue Volume in Adolescents and Adults with Autism", Biological Psychiatry Volume 59, Issue 1, 1 January 2006, Pages 1-6

13. Mousumi Bala, Suraiya Yasmin, "Study the Corpus Callosum of Brain to Explore Autism Employing Image Segmentation", International Journal of Neuroscience and Behavioral Science 4(3): 37-44, 2016 http://www.hrpub.org DOI: 10.13189/ijnbs.2016.040301

14. RussellLangabMarkO'ReillybOliveHealycMandyRispolidHelenaLydo ncWilliamStreusandeTonyaDavisfSoyeonKangbJeffSigafoosgGiulioLa ncionihRobertDiddeniSanneGiesbersi, "Sensory integration therapy for autism spectrum disorders: A systematic review", Research in Autism Spectrum Disorders Volume 6, Issue 3, July-September 2012, Pages 1004-1018.

15. JaganathanA.WeissSuzanneRobinsonStephanieFungAmiTintPhilipCha lmersYonaLunskyHartley,Seltzer,Head,\&Abbeduto, " Family hardiness, social support, and self efficacy in mothers of individual with autism spectrum disorders", Research in Autism, Elsevier publication, 2019 .

16. Ige-MarieEigstiaAshley B.de MarchenaaJillian .SchuhaElizabethKelleyb, “ Language acquisition in autism spectrum disorders: A developmental review", Research in Autism Spectrum Disorders Volume 5, Issue 2, April-June 2011, Pages 681-691

17. ArleneMannionGeraldineLeader, " Comorbidity in autism spectrum disorder: A literature review", Research in Autism Spectrum Disorders Volume 7, Issue 12, December 2013, Pages 1595-1616.

18. PhilippaMossa1, " Growing older with autism - The experiences of adult siblings of individuals with autism", Research in Autism Spectrum Disorders, Volume 63, July 2019, Pages 42-51

\section{AUTHORS PROFILE}

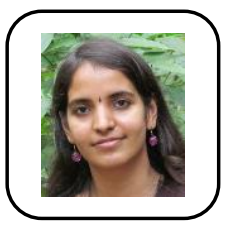

Nagashree N. working as Asst. Professor in Nagarjun College of Engineering \& Technology. She obtained B.E(CSE) in 2006 from VTU, and M.Tech (CSE) in 2010 and currently perusing Ph.D, She is having 8+ years of teaching experience, 1 year research experience at Indian Institute of Science, Bangalore. 6 National and International publications, Member of IEEE, Got best paper award in National Conference.

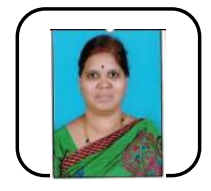

Dr. Premajyoti G. Patil working as Professor in Nagarjuna College of Engineering \& Technology. She obtained her BE degree in Instrumentation Technology from Gulbarga University, Gulbarga in 1994 and ME degree in Power electronics from Gulbarga University, Gulbarga in 1998 and $\mathrm{PhD}$ in Electrical \& Electronics Engineering in Dr. MGR Educational \& Research Institute University, Chennai. She is having 21 years of teaching experience \& she published twenty papers in National \& International conferences and guiding two PhD scholars. Her areas of interest are Microprocessors, Computer Networks, Computer Organization, Electronic Circuits, Logic Design. She has three best technical paper awards in her credit. She is recipient of state level project of the year award during the academic year 2015-2016 conducted by Karnataka State for Science and Technology. She worked as a resource person for summer Internship 2016 on IOT conducted by Texas Instruments University. She is Life member of ISTE and member of IEEE.

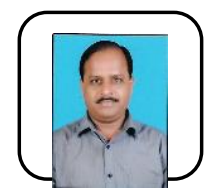

Dr. Shantakumar B Patil working as Professor in Nagarjuna College of Engineering \& Technology. He obtained his B.E degree in Electrical \& Electronics Engineering from Karnataka University Dharwad in 1993 and M.Tech in Computer Science \& Engineering from VTU Belagavi in 2002. He obtained Ph. D degree from Dr. MGR University, Chennai in 2011. His areas of interest are Data Mining, Artificial Intelligence, and Formal Languages \& Automata Theory. He has 24 years of experience in teaching and published twenty Research papers in National / International Journals and conferences. He is recipient of BEST TEACHER award twice, when he was in MVJ College of Engineering and has received BEST PAPER awards in National and International Conferences across the world. He is guiding five Research Scholars for Doctoral Degree. He is Life member of ISTE, CSI and member of IEEE.

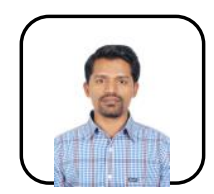

Mr. Mallikarjun Kokatanur, Working as Senio Software Engineer in Major IT company and he has obtained B.E(CSE) from VTU in 2006, and MS(IT) from Mangalore University in year 2012. And has 10 years of industrial experience in $\mathrm{C}, \mathrm{C}++$, Java, Python, Big Data, NoSQL. 